\title{
Structured reporting for fibrosing lung disease: a model shared by radiologist and pulmonologist
}

\author{
Nicola Sverzellati ${ }^{1} \cdot$ Anna Odone $^{2} \cdot$ Mario Silva $^{1} \cdot$ Roberta Polverosi $^{3} \cdot$ Carlo Florio $^{4} \cdot$ Luciano Cardinale $^{5}$. \\ Giancarlo Cortese $^{6}$. Giancarlo Addonisio ${ }^{3}$. Maurizio Zompatori ${ }^{7}$. Giorgia Dalpiaz ${ }^{8}$. Sara Piciucchi ${ }^{9}$. \\ Anna Rita Larici ${ }^{10}$. The Italian Structured Report on Fibrosing Lung Disease Consort
}

Received: 24 August 2017 / Accepted: 6 November 2017 / Published online: 11 December 2017

(c) The Author(s) 2017. This article is an open access publication

\begin{abstract}
Objectives To apply the Delphi exercise with iterative involvement of radiologists and pulmonologists with the aim of defining a structured reporting template for high-resolution computed tomography (HRCT) of patients with fibrosing lung disease (FLD).

Methods The writing committee selected the HRCT criteria - the Delphi items—for rating from both radiology panelists (RP) and pulmonology panelists (PP). The Delphi items were first rated by RPs as "essential", "optional", or "not relevant". The items rated "essential" by $<80 \%$ of the RP were selected for the PP rating. The format of reporting was rated by both RP and PP.

Results A total of 42 RPs and 12 PPs participated to the survey. In both Delphi round 1 and 2, 10/27 (37.7\%) items were rated "essential" by more than $80 \%$ of RP. The remaining 17/27 (63.3\%) items were rated by the PP in round 3, with 2/17 items (11.7\%) rated "essential" by the PP. PP proposed additional items for conclusion domain, which were rated by RPs in the fourth round. Poor consensus was observed for the format of reporting.

Conclusions This study provides a template for structured report of FLD that features essential items as agreed by expert thoracic radiologists and pulmonologists.
\end{abstract}

Keywords Structured report $\cdot$ Standardized report $\cdot$ Consensus $\cdot$ Lung fibrosis $\cdot$ High-resolution computed tomography

The Italian Structured Report on Fibrosing Lung Disease

Consort Collaborators are listed in Acknowledgements section.

Electronic supplementary material The online version of this article (https://doi.org/10.1007/s11547-017-0835-6) contains supplementary material, which is available to authorized users.

Nicola Sverzellati

nicolasve@tiscali.it

1 Section of Radiology, Unit of Surgical Sciences, Department of Medicine and Surgery (DiMeC), University of Parma, Parma, Italy

2 Department of Medicine and Surgery (DiMeC), University of Parma, Parma, Italy

3 Department of Radiology, Ospedali di San Donà di Piave e Jesolo, Padua, Italy

4 Department of Radiology, Istituto Tumori "Giovanni Paolo II" di Bari, Bari, Italy

\begin{abstract}
Abbreviation
DLD Diffuse lung disease

FLD Fibrosing lung disease

HRCT High-resolution computed tomography

NSIP Non-specific interstitial pneumonia

PPSs Pulmonology panelists

RPs Radiology panelists

UIP Usual interstitial pneumonia
\end{abstract}

5 Department of Radiology, S. Luigi Gonzaga Orbassano, Turin, Italy

6 Department of Radiology, Ospedale Maria Vittoria, Turin, Italy

7 Radiology, Policlinico S. Orsola-Malpighi, University of Bologna, Bologna, Italy

8 Department of Radiology, Azienda Unità Sanitaria Locale di Bologna, Bologna, Italy

9 Department of Radiology, GB Morgagni Hospital, Forlì, Italy

10 Department of Radiological Sciences, Institute of Radiology, Università Cattolica del Sacro Cuore, Rome, Italy 


\section{Introduction}

The radiology report is an essential part of the service that radiologists provide to both patients and referring physicians, in any field of medicine. It records information for future use and it is part of the legal records for the episode of care [1]. The radiology report's structure and content may vary according to several factors, including the clinical inquiry, and the radiologist's expertise and education.

Free text reporting is still the most common format in clinical radiology. However, free text clinical reports may heterogeneously render the core information (e.g., language and cultural variability), making it difficult to compare reports or find specific details [2,3]. This is particularly true for diffuse lung disease (DLD), which is often a challenging diagnosis and prone to variable description by high-resolution computed tomography (HRCT). Notably, there is no consensus on the relevant findings that should serve as core mandatory information to the referring physician. For instance, reporting enlarged lymphnodes might result misleading in patients with fibrosing lung disease (FLD) because these abnormalities frequently coexist without specific clinical implication.

A number of initiatives are being promoted by the major international societies of radiology to disseminate the use of structured reporting [4]. Potentially, the use of structured reporting may improve consistency in clinical radiology [5]. It might reduce the rate of overlooking important findings as well as improve communication with referring physician. Several studies have explored pros and cons of the structured reporting in various settings. Some of them showed some benefit whereas others did not [2, 5-7]. However, it seems that most radiologists are encouraging and appreciating the use of structured reporting, especially in among subspecialist radiologists [8].

To our knowledge, there is no proposed structured reporting template for HRCT scans of subjects with DLD to guide radiologists in the systematic reporting in the framework of findings and synthesize a final clinical hypothesis. Following debates between expert pulmonologists and chest radiologists at the Italian national meetings national meetings, respectively, from the Italian societies of respiratory medicine and the Italian society of medical radiology (SIRM), we hypothesized that a structured report might be particularly helpful for HRCT of patients with FLD.

Within the reference standard of multidisciplinary discussion, the radiology report remains the primary method of communication between radiologists and clinicians, particularly in non-referral centers. A simple stepwise approach can aid HRCT interpretation and is especially applicable to FLD, thus fitting structured reporting [9]. In fact, reducing the chance of omitting important findings (e.g., traction bronchiectasis or pulmonary emphysema), or supplying an interpretation of the radiological pattern, would be paramount to guide the referring physician in critical clinical decisions for patients with FLD.

This study was undertaken to enact critical shared discussion between chest radiologists and pulmonologists by means of multi-round consensus-building Delphi exercise, to develop a comprehensive focused structured reporting template for HRCT of patients with FLD. The study objectives were to develop a list of HRCT criteria to describe FLD, to learn the most relevant parameters according to the point of view of pulmonologist, and to assess the agreement among experts on the proposed criteria.

\section{Materials and methods}

A six members writing committee proposed the HRCT criteria and parameters-the Delphi items -to the panelists rating. The writing committee members had at least 10 years of experience and authored at least 10 studies in imaging of DLD and they did not participate in the following Delphi survey. The list of Delphi items was based on the evidence from the literature in FLD and the experience of each writing committee member.

\section{Selection of the Delphi domains and items}

A literature search was performed in Medline to identify publications relevant to the HRCT features of FLDs from 2002 idiopathic interstitial pneumonia classification document to January 2016 [10]. The full text of the selected studies was reviewed by two out of six members of the writing committee, who developed and shared the initial list of Delphi items with the other writing committee members via emails and teleconferences.

The structured report was divided into three domains according to the American College of Radiology handbook for residents: (a) initial considerations, (b) HRCT findings, (c) Conclusions [11]. The Delphi items proposed by the writing committee are detailed in Table 1 according to domain belonging.

The domain "Initial considerations" included information needed for pre-test description of the clinical scenario and the quality of HRCT, as follows: available clinical indication, HRCT technique details (including technical differences potentially affecting the study comparison), and disclosure of motion artifacts.

The domain "HRCT findings" included the comparison of the HRCT findings with prior examinations, notably referring to extent and type of radiological findings (e.g., reticular opacities are increased in extent, traction bronchiectasis look 
Table 1 List of Delphi items proposed by the writing committee for panelists survey

\begin{tabular}{|c|c|c|}
\hline Initial considerations & HRCT findings & Conclusions \\
\hline Available clinical indication & $\begin{array}{l}\text { Comparison of CT findings with prior scan, } \\
\text { indicating change of each CT finding }\end{array}$ & $\begin{array}{l}\text { CT pattern: } \\
\text { (1) DEFINITE UIP } \\
\text { (2) POSSIBLE UIP } \\
\text { (3) ACUTE COMPLICATIONS IN UIP } \\
\text { (4) NSIP } \\
\text { (5) NSIP-OP } \\
\text { (6) SARCOIDOSIS } \\
\text { (7) PPFE }\end{array}$ \\
\hline CT protocol details & $\begin{array}{l}\text { Initial assessment of signs of lung fibrosis } \\
\text { (honeycombing, traction bronchiectasis, signs } \\
\text { of volume loss) }\end{array}$ & Proposal for the subsequent diagnostic test \\
\hline Comparison with prior CT examinations & $\begin{array}{l}\text { Confidence on honeycombing against traction } \\
\text { bronchiectasis }\end{array}$ & Indication for the timing of CT follow-up \\
\hline $\begin{array}{l}\text { Differences in CT technique with prior examina- } \\
\text { tions }\end{array}$ & Avoid description of absent findings & \\
\hline \multirow[t]{15}{*}{ Motion artifacts } & Description of all CT findings & \\
\hline & $\begin{array}{l}\text { Description of the most relevant CT findings } \\
\text { only (e.g., honeycombing, traction bronchiecta- } \\
\text { sis, signs of volume loss) }\end{array}$ & \\
\hline & $\begin{array}{l}\text { Disease distribution on both axial and cranio- } \\
\text { caudal planes }\end{array}$ & \\
\hline & $\begin{array}{l}\text { Differentiation between macro- and micro-cystic } \\
\text { honeycombing }\end{array}$ & \\
\hline & $\begin{array}{l}\text { Description of the reticular opacities subtypes } \\
\text { (e.g., intralobular or interlobular) }\end{array}$ & \\
\hline & $\begin{array}{l}\text { Emphysema subtype, including the so-called } \\
\text { airspace enlargement with fibrosis }\end{array}$ & \\
\hline & $\begin{array}{l}\text { Quantitation of FLD extent as percentage of the } \\
\text { lung volume }\end{array}$ & \\
\hline & $\begin{array}{l}\text { Quantitation of FLD extent according to three } \\
\text { categories of severity }\end{array}$ & \\
\hline & $\begin{array}{l}\text { Quantitation of FLD extent for any disease OR } \\
\text { for sarcoidosis and systemic sclerosis only }\end{array}$ & \\
\hline & $\begin{array}{l}\text { Quantitation of emphysema extent as percentage } \\
\text { of the lung volume }\end{array}$ & \\
\hline & $\begin{array}{l}\text { Quantitation of emphysema extent according to } \\
\text { three categories of severity }\end{array}$ & \\
\hline & $\begin{array}{l}\text { Report air trapping only when expiratory CT } \\
\text { scan is performed }\end{array}$ & \\
\hline & Suggest air trapping also on inspiratory CT scan & \\
\hline & $\begin{array}{l}\text { Report enlarged pulmonary artery for any dis- } \\
\text { ease OR for sarcoidosis and systemic sclerosis } \\
\text { only }\end{array}$ & \\
\hline & Report enlarged lymph-nodes & \\
\hline
\end{tabular}

$O P$ organizing pneumonia; $P P F E$ Pleuro-Parenchymal Fibroelastosis

more severe, ground-glass opacity resolved, ground-glass opacity evolved towards overt reticulation, etc.). Furthermore, hierarchy of HRCT findings description was investigated with particular emphasis on the key components of the FLD, namely honeycombing, traction bronchiectasis, and signs of volume loss [9]. Furthermore, it was asked whether the description of the HRCT findings had to be concise or include also missing finding (e.g., not signs of honeycombing, not air trapping, etc.). It was also investigated the relevance of disclosure of the confidence in diagnosing and differentiating honeycombing and traction bronchiectasis, which is a critical task even among expert chest radiologists [12].

Quantitative information was also among the items of "HRCT findings", namely the relevance of reporting the extent of fibrosis and emphysema in the structured report. 
Furthermore, the format of preferred visual scoring was proposed as either continuous variable (e.g., percentage) or as category (e.g., mild, moderate, or severe). Of note, no cut-off values were a priori set to define the categories of disease extent (e.g., the category should be assigned according to radiologist subjective impression). In addition, it was investigated whether extent should be provided for any FLD or only for individual disorders (e.g., sarcoidosis, systemic sclerosis) for which literature supported the prognostic value of such data at the time of the present study [13, 14]. Scientific references were supplied to the panelists for informed review of the aforementioned items.

The domain "Conclusions" was meant to investigate the relevance of reporting the HRCT pattern (e.g., usual interstitial pneumonia, UIP; non-specific interstitial pneumonia, NSIP, etc.), of proposing further diagnostic evaluation, and of indicating the timing for HRCT follow-up.

\section{Selection of the study panelists}

The Italian Chest Imaging Subspecialty Society indicated a list of 53 expert radiologists ( 38 males, age range 30-72 years, median age 43 years, median years of experience in DLD 11 years), all active members of the Section of Thoracic Imaging of the SIRM, to participate in the Delphi survey (i.e., the radiology panelists-RPs). Selection criteria for RPs were as follows: (a) $\geq 5$ years of experience in imaging of DLD (as reported in the society database); (b) authorship of $\geq 3$ articles on DLD in peer-reviewed journal. Likewise, 18 pulmonologists (13 males, age range 38-61 years, median age 53 years, median years of experience in DLD 15) panelist (PP) were selected according to the following selection criteria: (a) $\geq 10$ years of experience in DLD; (b) authorship of $\geq 10$ articles on DLD in peer-reviewed journal. The selected RPs and PPs were contacted by email, informed about the aims and methodology of the study.

\section{Overview of the Delphi exercise}

The Delphi exercise was run by a biostatistician (with 5 years of experience), through emails exchange.

First, RPs were asked to classify Delphi items into three categories, as follows: (a) "essential"; (b) "optional"; or (c) "not relevant" for the HRCT structured reporting. Items rated "essential" were further classified according to the format of reporting into "free text" or "outlined" (i.e., by a fixed check-list of descriptors or categories). A threshold of $80 \%$ "essential" rating by the RPs was set to retain items in the final structured report. Thereafter, items rated "essential" by less than $80 \%$ of the RPs were subsequently used to compile the Delphi item list for the PPs round. Again, a threshold of $80 \%$ "essential" rating by the PPs was set to retain items in the definite structured report. Items rated "not relevant" were considered useless or misleading, therefore, excluded from the final structured report. Furthermore, all panelists were allowed to suggest additional items to be included in the item list for the subsequent round; this fostered the open discussion that this inter-specialty consultation was meant for.

In addition, PPs were also asked to judge the Delphi items that had been rated "essential" by the RPs, as follows: agree or disagree. The detailed sequence of steps of the Delphi survey is shown in Fig. 1.

\section{Data analysis}

Survey analysis was prospectively performed round by round [15]. In particular, the results of round 1 provided the content for sequential surveys. The cut-off value to retain an item within the exercise and eventually in the HRCT reporting model was $\geq 80 \%$ "essential" rating $[16,17]$. Items with $<80 \%$ "essential" rating were iteratively rated at subsequent rounds. Likewise, this cut-off value was also used to select the most appropriate format of reporting of each retained item (e.g., to be given as free text or to be outlined).

\section{Results}

Four Delphi rounds were performed to obtain the final structured report. A total of 42/53 (79.2\%) invited radiologists agreed to participate as RPs. All of them completed the first round, $41(97.6 \%)$ and $36(85.7 \%)$ completed the second and the fourth round, respectively. A total of 12/18 (66.7\%) pulmonologists agreed to participate as PPs and completed one Delphi round (i.e., the third round).

The result of each round is given in supplementary Tables 1-4 and summarized in Fig. 1. In round 1, 10/27 (37.7\%) Delphi items were rated "essential" by more than $80 \%$ of RPs. In particular, total or nearly total agreement was reached for the following items: presence of prior HRCT scans for comparison, initial description of the variation of HRCT findings as compared to prior scans, and assessment of the disease distribution. No format option reached the cut-off of $80 \%$ and was re-rated in round 2 by the RPs. A number of panelists expressed no preference for the format of reporting as outlined in supplementary Tables.

In round 2, 17/27 (63\%) items were re-rated by the RPs. A $80 \%$ consensus was not reached for any of them. A consensus greater than $80 \%$ was recorded upon the outline format for four out of $10(40 \%)$ items that had been judged "essential" in round 1 . Ten out of $42(24 \%)$ RPs suggested to add an item for ancillary findings (e.g., pleural plaques, dilated esophagus, etc.), and report it as "free text".

In round 3, PPs rated "essential" the extent of the visual scoring of both FLD (expressed as percentage lung volume involvement) and emphysema (expressed as categories). No 


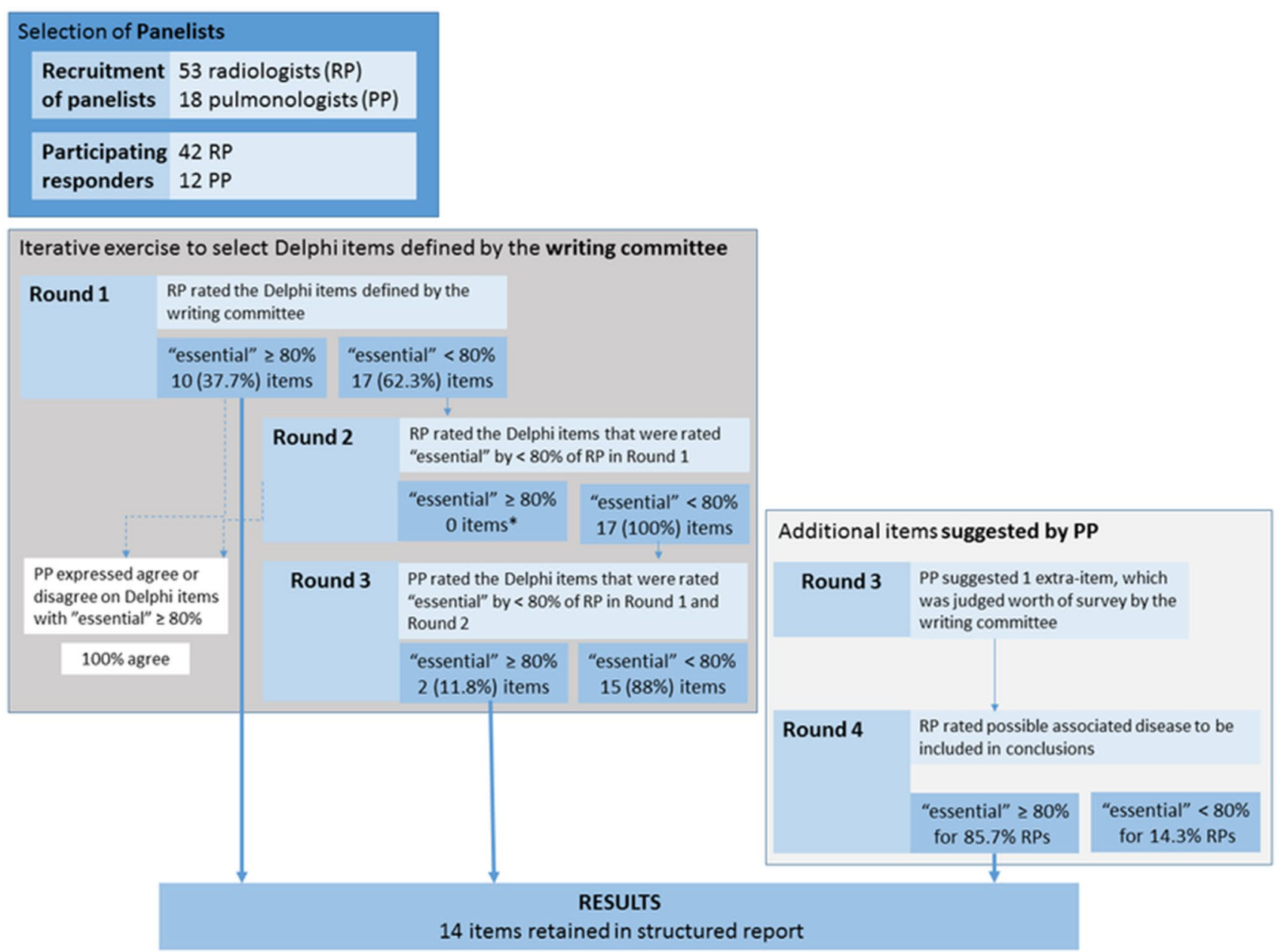

Fig. 1 Diagram summarizing the Delphi rounds results

sufficient consensus was reached for the remaining 15 items that had not been rated "essential" by the RPs. Besides, the PPs unanimously agreed on both the set of 10 "essential" items chosen by the RPs. Furthermore, three (25\%) PPs suggested to expand on the conclusions domain by including a more detailed list of HRCT patterns and potential association or cause (e.g., unknown connective tissue disease, asbestosis, etc.) of FLD predictable from the HRCT findings. This was regarded as worth of rating by the radiologists writing committee that agreed upon a list of both HRCT patterns and associations of FLD.

In round 4, RPs chose between including only the HRCT patterns list or also suggesting any association or cause of the FLD in the structured report. There was substantial agreement upon suggesting the association or cause of the FLD too ( $85.7 \%$ "essential").

The format option reached $80 \%$ consensus or higher for a few Delphi items (supplementary Tables 1-4). The format of each item included in the final template was chosen by the writing committee by taking into account the preferences of the majority of the RPs and PPs. Likewise, given the lack of consensus on the description of the CT findings other than CT features of FLD and the suggestion of reporting ancillary findings in round 2, an item "other CT findings" (to be reported as "free text") was included in the final structured report template.

By accounting for the rounds results, a final structured report template was finally developed by the writing committee, circulated and approved among all the participants (Fig. 2).

\section{Discussion}

We applied a rigorous consensus-building technique to identify the key items to be included in a structured report of FLD. In particular, it is the result of joint discussion between thoracic radiologists and pulmonologists. The iterative structure of the Delphi exercise showed a substantial agreement on the items progressively proposed for inclusion by each 


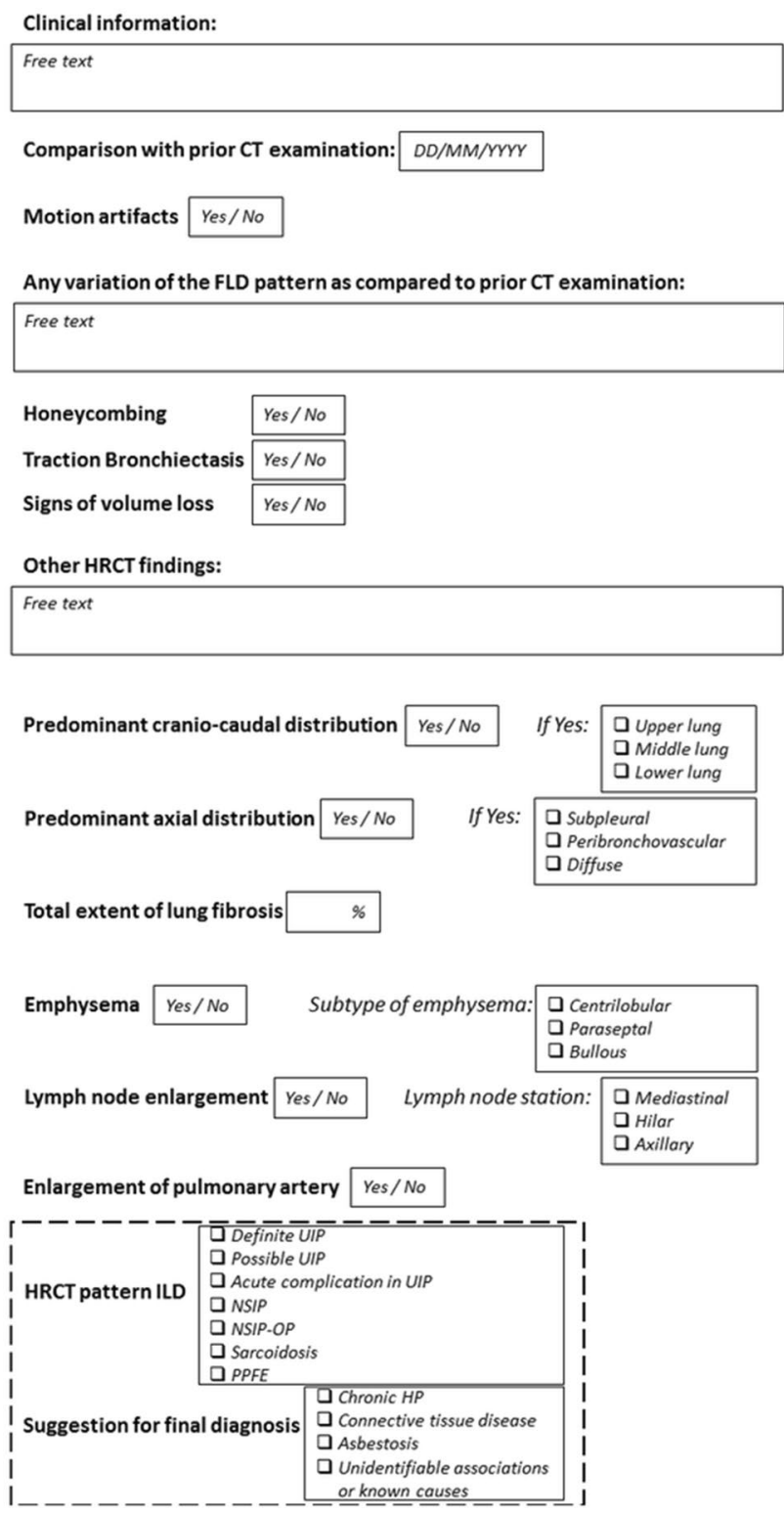

Fig. 2 Final structured report template approved by all the study participants

board of panelists, at subsequent rounds. The proposed template for structured report of FLD comes as the first multidisciplinary example in the field.

The glossary of terms for thoracic imaging as well as the classification documents have both improved the interpretation of the FLD [18-20]. However, there is no official reference for consistent reporting HRCT scan of subjects with FLD. This deficiency may have an impact on medical practice. Noteworthy, standardized structured reporting may reduce the risk of under-reporting important findings of FLD, especially in peripheral centers. Therefore, as for other specific topics, a structured report may well fit the FLD [21].
Ideally, the radiologic report should address both clinical and radiological needs, with a direct and easy format. On the clinical side, there is no official recommendation about the mandatory features of a radiological report, notably about suggesting association between HRCT pattern and cause of DLD. Furthermore, it is not clear whether the radiologic report should include proposals for the management. Both topics should be discussed according to the potential influence on clinical decision making, even in terms of potential medico-legal consequence [22]. This study was indeed driven by the need for practical interaction to improve the usefulness of the basic communicating system within multidisciplinary management of the patient, namely the radiologic report. Therefore, pulmonologists were involved in this study to integrate their needs in a shared structured report, and to rate the relevance of items selected by radiologists.

Interestingly, pulmonologists agreed on the choice of radiologists and suggested to include information on the extent of both FLD and emphysema, which were not rated "essential" by a sufficient proportion of radiologists. Furthermore, pulmonologists proposed an additional item for the conclusions, namely the suggestion of possible cause or association of the FLD, which might be particularly useful to define the clinical choice for subsequent diagnostic work-up or management. For example, the compulsory radiological suggestion of a potential cause of FLD (e.g., asbestosis, connective tissue disease, etc.) rather than the sole HRCT pattern (e.g., UIP or NSIP), might encourage both radiologists to systematically look for important ancillary findings (e.g., pleural plaques, dilated esophagus, etc.) and pulmonologists to seek clinical investigation for the proposed association [23].

The Delphi exercise is one of the strengths of the present study. Indeed, it is a widely recognized method to investigate consensus among experts. Noteworthy, the anonymity of panelists reduces the potential influence of strong opinion leaders on other participants and, thereby, the results of the survey [24]. The specific design of our Delphi survey was meant to first gather the opinion of radiologists, and then to investigate agreement of pulmonologists, especially on topics that are essential to the majority of expert radiologists. This logical sequence was particularly important for technical and medico-legal issues, which may be beyond the radiological "know how" of pulmonologists. Therefore, this approach granted main radiological address of the report with clinically significant adjustment from pulmonologists. The unanimous agreement of pulmonologists on items retained by radiologists suggests that all those items were clinically necessary, but allegedly they were not sufficient.

There are multiple formats for structured report, with no one being absolutely preferable over the other. They range from free text reporting with reference headers to predefined point-and-click options [25]. In our study, the panelists were 
asked to choose their preferred format for each item. There was poor consensus (threshold for consensus $\geq 80 \%$ ) on the format for structured report. Both radiologists and pulmonologists did not consider essential the reporting style of HRCT findings, particularly the avoidance of over-description and the disclosure of confidence on interpretation of key HRCT findings (e.g., honeycombing vs reticular abnormalities superimposed to emphysema). Therefore, the format of each item included in the final template was chosen by the writing committee by taking into account the preferences of the majority of the RPs and PPs. We think that a combination of free text and outlined items may be helpful to either systematically disclose key unequivocal HRCT features (e.g., honeycombing) or describe the meaning of individual abnormalities that otherwise would be difficult to capture using a fixed check-list.

Further items included in the final structured report template are worth of discussion. The clinical inquiry should be included in the first rows of the report to facilitate the structured answer to the specific question. Conversely, stating the absence of pertinent clinical history may help convey diagnostic uncertainties [25]. Moreover, the comparison with prior examinations might be prognostically relevant, thus it should be clearly addressed at each HRCT control. The proposed structured report encourages the key discrimination between FLD and non-fibrotic DLD. Notably, the structured description of HRCT signs of FLD allows the list of potential diagnoses of DLD to be narrowed from over a hundred disorders to a handful of diseases [9].

Radiologists did not reach a consensus on the format of preferred visual scoring for emphysema extent. This was probably due to the current lack of clarity about the best method to be applied for that purpose, indeed the visual score is affected by quite an inter-observer variation. Furthermore, the utility of semi-automatic software for emphysema quantitation in routine activity has yet to be clarified, especially for emphysema in FLD [26]. Density masking is the current reference standard for emphysema quantification. This method is not suitable for quantification of patients with emphysema and FLD because it cannot discriminate between low density areas due to emphysema or associated with honeycomb cysts or traction bronchiectasis [27]. On the other hand, the total disease extent by categories would help stratifying patient prognosis, especially in sarcoidosis and systemic sclerosis. This was a topic of debate between radiologists and pulmonologists. In our survey, the radiologists did not reach a consensus on that $[13,14]$, whilst the pulmonologists preferred to include specific description of disease extent for any FLD as well as emphysema. This request from the pulmonologists is likely related to the need to get additional instruments to improve their interpretation of the patient clinico-functional profile.
The inclusion of air trapping in FLD seemed also concerning for structure report. The predictive value of air trapping in FLD is still under evaluation, with no cut-off extent being recognized. Furthermore, air trapping may be independent from specific association with FLD (e.g., it has been reported in up to $32 \%$ of IPF cases) [28]. In a recent study, diffuse air trapping was the source of CT-pathologic discordance in $71.8 \%$ in IPF [29].

In the "Conclusions" domain, neither radiologists nor pulmonologists agreed upon suggesting specific diagnostic work-up because it was likely perceived as potentially misleading, allegedly beyond the radiologists knowledge (e.g., radiologists have often limited information about the patient clinical condition and indeed cannot figure out what would be best for him).

This study has some limitations. First, the study panelists were from a single country, for this reason there was a relatively small number of selected expert panelists. The participation of opinion leaders from multiple countries would grant broader sharing and would increase the consistency of the structured report. We foster this National survey will prompt international survey to increase the number of participants, which was likely not sufficiently large to address the study task. The Delphi items were evaluated according to the Italian terminology, nevertheless international scientific references were supplied for informed review. Second, the items were initially selected by the study writing committee, thus resulting in a modified Delphi exercise that could potentially bias the structured report outline. Finally, this study did not aim to assess the impact of the structured report in the diagnosis and management of FLD. This issue will be discussed by forthcoming studies.

In conclusion, this study provides a template for structured report of FLD that features essential items as agreed by thoracic radiologists and their main speaker, the pulmonologists. The multidisciplinary methodology strengthens the structured report utility for daily practice.

Acknowledgements Carlo Agostini ${ }^{1}$, Carlo Albera ${ }^{2}$, Domenico Attinà $^{3}$, Giuseppe Battista ${ }^{4}$, Elena Bertelli ${ }^{5}$, Giuseppina Bertorelli ${ }^{6}$, Claudio Bnà ${ }^{7}$, Martina Bonifazi ${ }^{8}$, Lorenzo Bonomo ${ }^{9}$, Andrea Borghesi ${ }^{10}$, Lucio Calandriello ${ }^{9}$, Antonella Caminati ${ }^{11}$, Diana Capannelli ${ }^{3}$, Stefania Cerri ${ }^{12}$, Federica Ciccarese ${ }^{3}$, Davide Colombi ${ }^{13}$, Marco Confalonieri ${ }^{14}$, Annaemilia Del Ciello ${ }^{9}$, Giovanni Della Casa ${ }^{15}$, Roberto Dore ${ }^{16}$, Fabio Falaschi ${ }^{17}$, Alessandra Farchione ${ }^{9}$, Beatrice Feragalli $^{18}$, Paola Franchi ${ }^{9}$, Giampaolo Gavelli ${ }^{19}$, Sergio Harari ${ }^{11}$, Fabrizio Luppi ${ }^{12}$, Fabio Maggi ${ }^{20}$, Maria Antonietta Mazzei ${ }^{21}$, Manuela Mereu $^{22}$, Gianluca Milanese ${ }^{23}$, Stefano Palmucci ${ }^{24}$, Rosa Lucia Patea ${ }^{22}$, Alberto Pesci ${ }^{25}$, Marco Piolanti ${ }^{26}$, Venerino Poletti ${ }^{27}$, Gaetano Rea ${ }^{28}$, Luca Richeldi $^{29}$, Paola Rogliani ${ }^{30}$, Chiara Romei ${ }^{17}$, Paola Rottoli ${ }^{31}$, Alessandro Sanduzzi-Zamparelli ${ }^{32}$, Alfredo Sebastiani ${ }^{33}$, Gianluigi Sergiacomi ${ }^{34}$, Gian Alberto Soardi ${ }^{35}$, Lucia Spaggiari ${ }^{36}$, Paolo Spagnolo $^{37}$, Sara Tomassetti ${ }^{27}$, Rocco Trisolini ${ }^{38}$, Adele Valentini ${ }^{16}$, Carlo Vancheri ${ }^{39}$, Valentina Vespro ${ }^{40}$, Luca Volterrani ${ }^{21}$

${ }^{1}$ Clinical Immunology, Department of Medicine (DIMED), Padua University, Padua, Italy; ${ }^{2}$ Department of Clinical and Biological 
Sciences, University of Turin, Turin, Italy; ${ }^{3}$ Radiology Unit, CardioThoracic-Vascular University Hospital S. Orsola-Malpighi, Bologna, Italy; ${ }^{4}$ Dipartimento di Medicina Specialistica, Diagnostica e Sperimentale, University Hospital S. Orsola-Malpighi, Bologna, Italy; ${ }^{5}$ Radiologia, Ospedale Careggi, Firenze, Italy; ${ }^{6}$ Respiratory Disease and Lung Function Unit, Department of Medicine and Surgery, University of Parma, Italy; ${ }^{7}$ Radiology, Poliambulanza Foundation Hospital, Brescia, Italy; ${ }^{8}$ Department of Biomedical Sciences and Public Health, Università Politecnica delle Marche, Ancona, Italy; ${ }^{9}$ Institute of Radiology, Department of Radiological Sciences, Università Cattolica del Sacro Cuore, Rome, Italy; ${ }^{10}$ Department of Radiology, University and Spedali Civili of Brescia, Italy; ${ }^{11}$ U.O. di Pneumologia e Terapia Semi-Intensiva Respiratoria - Servizio di Fisiopatologia Respiratoria ed Emodinamica Polmonare, Ospedale San Giuseppe - MultiMedica IRCCS, Milan, Italy; ${ }^{12}$ Center for Rare Lung Diseases, University Hospital Policlinico di Modena, Italy; ${ }^{13}$ Department of Radiology, Guglielmo da Saliceto Hospital, Piacenza, Italy; ${ }^{14}$ Pulmonology Department, University Hospital of Cattinara, Trieste, Italy; ${ }^{15}$ Radiology, University Hospital Policlinico di Modena, Italy; ${ }^{16}$ Unit of Radiology, IRCCS Policlinico San Matteo, Pavia, Italy; ${ }^{17}$ Second Radiology Unit, University Hospital of Pisa, Pisa, Italy; ${ }^{18}$ Department of Medical, Oral and Biotechnological Sciences, University G. D'Annunzio, Chieti, Italy; ${ }^{19}$ Radiology Unit, IRCCS Istituto Scientifico Romagnolo per lo Studio e la Cura dei Tumori (IRST), Meldola (FC), Italy; ${ }^{20}$ Azienda sanitaria locale n. 1, Avezzano, Sulmona, L'Aquila, Italy; ${ }^{21}$ Department of Medical, Surgical and Neuro Sciences, Diagnostic Imaging, Azienda Ospedaliera Universitaria Senese, University of Siena, Siena, Italy; ${ }^{22}$ Department of Neuroscience and Section of Integrated Imaging and Radiological Therapies, University of Chieti, Italy; ${ }^{23}$ Section of Radiology, Unit of Surgical Sciences, Department of Medicine and Surgery (DiMeC), University of Parma, Italy; ${ }^{24}$ Department of Medical and Surgical Sciences and Advanced Technologies-Radiodiagnostic and Radiotherapy Unit, University Hospital "Policlinico-Vittorio Emanuele", Catania, Italy; ${ }^{25}$ Department of Health Science, Pulmonology Unit, University of Milan Bicocca, San Gerardo Hospital, Monza, Italy; ${ }^{26}$ Radiologia, Azienda Unità Sanitaria Locale di Bologna, Italy; ${ }^{27}$ Department of Diseases of the Thorax, Azienda USL Romagna, GB Morgagni Hospital, Forlì, Italy; ${ }^{28}$ Department of Diagnostic Imaging, Monaldi Hospital, Naples, Italy; ${ }^{29}$ Unità Operativa Complessa di Pneumologia, Università Cattolica del Sacro Cuore, Fondazione Policlinico A. Gemelli, Rome, Italy; ${ }^{30}$ University of Rome Tor Vergata, Department of Systems Medicine, Rome, Italy; ${ }^{31}$ Department of Medical and Surgical Sciences and Neurosciences, Respiratory Diseases Unit, University of Siena, Italy; ${ }^{32}$ Respiratory Medicine Section, Department of Clinical Medicine and Surgery, "Federico II" University of Naples, Naples, Campania, Italy; ${ }^{33}$ Interstiziopatie Polmonari Dipartimento Medico-Chirurgico dei percorsi integrati. Azienda Ospedaliera San Camillo-Forlanini di Roma, Italy; ${ }^{34}$ Department of Diagnostic and Molecular Imaging, Radiation Therapy and Interventional Radiology, University Hospital Tor Vergata, Rome, Italy; ${ }^{35}$ UOC Radiologia, Ospedale Maggiore di Borgo Trento, AOUI Verona, Verona, Italy; ${ }^{36}$ Radiologia, Arcispedale S. Maria Nuova - Azienda-Ospedaliera di Reggio Emilia, Italy; ${ }^{37}$ Section of Respiratory Diseases, Department of Cardiac, Thoracic, and Vascular Sciences, University of Padua, Padua, Italy; ${ }^{38}$ Interventional Pulmonology Unit, Policlinico S. OrsolaMalpighi, Bologna, Italy; ${ }^{39}$ Department of Clinical and Experimental Medicine, Regional Centre for Interstitial and Rare Lung Diseases, University of Catania, Catania, Italy; ${ }^{40}$ Dipartimento di Diagnostica per Immagini, UOC di Radiologia, Fondazione IRCCS Ca' Granda, Ospedale Maggiore Policlinico, Milan, Italy.

\section{Compliance with Ethical Standards}

Conflict of interest The authors state that this study was not funded. Dr. Albera reports grants, personal fees and non-financial support from
Roche, during the conduct of the study; personal fees and non-financial support from Roche, personal fees and non-financial support from Boehringer Ingelheim, personal fees and non-financial support from Fibrogen, personal fees and non-financial support from GSK, personal fees and non-financial support from Sanofi Aventis, outside the submitted work. Dr. Harari reports personal fees from Roche, grants and personal fees from Intermune, grants and personal fees from Boehringer Ingelheim, outside the submitted work. Dr. Luppi reports personal fees from Boehringer Ingelheim, grants and personal fees from Roche, during the conduct of the study. Dr. Odone reports personal fees from Roche, during the conduct of the study. Dr. Silva reports personal fees from Roche, outside the submitted work. Dr. Sverzellati reports personal fees from Roche, personal fees from Boehringer Ingelheim, outside the submitted work. The other authors declare that they have no conflict of interest.

Ethical approval The manuscript does not contain clinical studies or patient data.

Open Access This article is distributed under the terms of the Creative Commons Attribution 4.0 International License (http://creativecommons.org/licenses/by/4.0/), which permits unrestricted use, distribution, and reproduction in any medium, provided you give appropriate credit to the original author(s) and the source, provide a link to the Creative Commons license, and indicate if changes were made.

\section{References}

1. http://www.radreport.org/about.php

2. Schwartz LH, Panicek DM, Berk AR, Li Y, Hricak H (2011) Improving communication of diagnostic radiology findings through structured reporting. Radiology 260(1):174-181. https:// doi.org/10.1148/radiol.11101913

3. Reiner BI (2009) The challenges, opportunities, and imperative of structured reporting in medical imaging. J Digit Imaging 22(6):562-568. https://doi.org/10.1007/s10278-009-9239-z

4. European Society of R (2011) Good practice for radiological reporting. Guidelines from the European Society of Radiology (ESR). Insights Imaging 2(2):93-96. https://doi.org/10.1007/ s13244-011-0066-7

5. Larson DB, Towbin AJ, Pryor RM, Donnelly LF (2013) Improving consistency in radiology reporting through the use of department-wide standardized structured reporting. Radiology 267(1):240-250. https://doi.org/10.1148/radiol.12121502

6. Andreisek G, Deyo RA, Jarvik JG, Porchet F, Winklhofer SF, Steurer J, group Lw (2014) Consensus conference on core radiological parameters to describe lumbar stenosis-an initiative for structured reporting. Eur Radiol 24(12):3224-3232. https://doi. org/10.1007/s00330-014-3346-Z

7. Mamisch N, Brumann M, Hodler J, Held U, Brunner F, Steurer J, Lumbar Spinal Stenosis Outcome Study Working Group Z (2012) Radiologic criteria for the diagnosis of spinal stenosis: results of a Delphi survey. Radiology 264(1):174-179. https:// doi.org/10.1148/radiol.12111930

8. Powell DK, Silberzweig JE (2015) State of structured reporting in radiology, a survey. Acad Radiol 22(2):226-233. https://doi. org/10.1016/j.acra.2014.08.014

9. Jacob J, Hansell DM (2015) HRCT of fibrosing lung disease. Respirology. https://doi.org/10.1111/resp.12531

10. American Thoracic S, European Respiratory S (2002) American Thoracic Society/European Respiratory Society International Multidisciplinary Consensus Classification of the Idiopathic Interstitial Pneumonias. This joint statement of the American Thoracic 
Society (ATS), and the European Respiratory Society (ERS) was adopted by the ATS board of directors, June 2001 and by the ERS Executive Committee, June 2001. Am J Respir Crit Care Med 165(2):277-304. https://doi.org/10.1164/ajrccm.165.2.ats01

11. DeQuesada ICZ, Cizman Z, Hill P, Wang W (eds) (2015) Getting started: a guide to year one of radiology residency. ACR resident and fellow section, 2nd edn. Available at: https://www. acr.org/ /media/ACR/Documents/PDF/Membership/RFS/2015 RFS_Guide_Final.pdf?la $=$ en

12. Watadani T, Sakai F, Johkoh T, Noma S, Akira M, Fujimoto K, Bankier AA, Lee KS, Muller NL, Song JW, Park JS, Lynch DA, Hansell DM, Remy-Jardin M, Franquet T, Sugiyama Y (2013) Interobserver variability in the CT assessment of honeycombing in the lungs. Radiology 266(3):936-944. https://doi.org/10.1148/ radiol.12112516

13. Goh NS, Desai SR, Veeraraghavan S, Hansell DM, Copley SJ, Maher TM, Corte TJ, Sander CR, Ratoff J, Devaraj A, Bozovic G, Denton CP, Black CM, du Bois RM, Wells AU (2008) Interstitial lung disease in systemic sclerosis: a simple staging system. Am J Respir Crit Care Med 177(11):1248-1254. https://doi. org/10.1164/rccm.200706-8770C

14. Walsh SL, Wells AU, Sverzellati N, Keir GJ, Calandriello L, Antoniou KM, Copley SJ, Devaraj A, Maher TM, Renzoni E, Nicholson AG, Hansell DM (2014) An integrated clinicoradiological staging system for pulmonary sarcoidosis: a case-cohort study. Lancet Respir Med 2(2):123-130. https://doi.org/10.1016/ S2213-2600(13)70276-5

15. Hsu CC, Sandford BA (2007) The Delphi technique: making sense of consensus. Pract Assess Res Eval 12(10):1531-7714

16. Fink A, Kosecoff J, Chassin M, Brook RH (1984) Consensus methods: characteristics and guidelines for use. Am J Public Health 74(9):979-983

17. Hsu CC, Sandford BA (2007) The Delphi technique: making sense of consensus. Pract Assess Res Eval 12(10):1-8

18. Hansell DM, Bankier AA, MacMahon H, McLoud TC, Muller NL, Remy J (2008) Fleischner Society: glossary of terms for thoracic imaging. Radiology 246(3):697-722. https://doi. org/10.1148/radiol.2462070712

19. Raghu G, Collard HR, Egan JJ, Martinez FJ, Behr J, Brown KK, Colby TV, Cordier JF, Flaherty KR, Lasky JA, Lynch DA, Ryu JH, Swigris JJ, Wells AU, Ancochea J, Bouros D, Carvalho C, Costabel U, Ebina M, Hansell DM, Johkoh T, Kim DS, King TE Jr, Kondoh Y, Myers J, Muller NL, Nicholson AG, Richeldi L, Selman M, Dudden RF, Griss BS, Protzko SL, Schunemann HJ, Fibrosis AEJACoIP (2011) An official ATS/ERS/JRS/ALAT statement: idiopathic pulmonary fibrosis: evidence-based guidelines for diagnosis and management. Am J Respir Crit Care Med 183(6):788-824. https://doi.org/10.1164/rccm.2009-040GL

20. Travis WD, Costabel U, Hansell DM, King TE Jr, Lynch DA, Nicholson AG, Ryerson CJ, Ryu JH, Selman M, Wells AU, Behr
J, Bouros D, Brown KK, Colby TV, Collard HR, Cordeiro CR, Cottin V, Crestani B, Drent M, Dudden RF, Egan J, Flaherty K, Hogaboam C, Inoue Y, Johkoh T, Kim DS, Kitaichi M, Loyd J, Martinez FJ, Myers J, Protzko S, Raghu G, Richeldi L, Sverzellati N, Swigris J, Valeyre D, Pneumonias AECoII (2013) An official American Thoracic Society/European Respiratory Society statement: update of the international multidisciplinary classification of the idiopathic interstitial pneumonias. Am J Respir Crit Care Med 188(6):733-748. https://doi.org/10.1164/ rccm.201308-1483ST

21. Gunderman RB, McNeive LR (2014) Is structured reporting the answer? Radiology 273(1):7-9. https://doi.org/10.1148/ radiol.14132795

22. Lafortune M, Breton G, Baudouin JL (1988) The radiological report: what is useful for the referring physician? Can Assoc Radiol J 39(2):140-143

23. Sverzellati N, Lynch DA, Hansell DM, Johkoh T, King TE Jr, Travis WD (2015) American Thoracic Society-European Respiratory Society Classification of the Idiopathic Interstitial Pneumonias: advances in Knowledge since 2002. Radiographics 35(7):1849-1871. https://doi.org/10.1148/rg.2015140334

24. Milholland AV, Wheeler SG, Heieck JJ (1973) Medical assessment by a Delphi group opinion technic. N Engl J Med 288(24):12721275. https://doi.org/10.1056/NEJM197306142882405

25. Wallis A, McCoubrie P (2011) The radiology report-are we getting the message across? Clin Radiol 66(11):1015-1022. https:// doi.org/10.1016/j.crad.2011.05.013

26. Mascalchi M, Diciotti S, Sverzellati N, Camiciottoli G, Ciccotosto C, Falaschi F, Zompatori M (2012) Low agreement of visual rating for detailed quantification of pulmonary emphysema in whole-lung CT. Acta Radiol 53(1):53-60. https://doi.org/10.1258/ ar.2011.110419

27. Hansell DM, Goldin JG, King TE Jr, Lynch DA, Richeldi L, Wells AU (2015) CT staging and monitoring of fibrotic interstitial lung diseases in clinical practice and treatment trials: a position paper from the Fleischner Society. Lancet Respir Med 3(6):483-496. https://doi.org/10.1016/S2213-2600(15)00096-X

28. Silva CI, Muller NL, Lynch DA, Curran-Everett D, Brown KK, Lee KS, Chung MP, Churg A (2008) Chronic hypersensitivity pneumonitis: differentiation from idiopathic pulmonary fibrosis and nonspecific interstitial pneumonia by using thin-section CT. Radiology 246(1):288-297. https://doi.org/10.1148/ radiol.2453061881

29. Yagihashi K, Huckleberry J, Colby TV, Tazelaar HD, Zach J, Sundaram B, Pipavath S, Schwarz MI, Lynch DA, Idiopathic Pulmonary Fibrosis Clinical Research N (2016) Radiologic-pathologic discordance in biopsy-proven usual interstitial pneumonia. Eur Respir J 47(4):1189-1197. https://doi. org/10.1183/13993003.01680-2015 